\title{
Nivel de implicancias físicas y emocionales en el profesional de Enfermería frente a la indicación médica del retiro de métodos de soporte vital en el paciente terminal, de la Unidad de Cuidados Intensivos del "Instituto Nacional Materno Perinatal” y el "Hospital Central de la Fuerza Aérea del Perú”, 2009.
}

\author{
Level of physical and emotional implications for nurse medical indication versus withdrawal of \\ life support methods in the terminal patient, the Intensive Care Unit "National Maternal Perinatal \\ Institute" and the "Central Hospital of the Air Force of Peru", 2009.
}

\begin{abstract}
Irene Beatriz Espinoza de la Sota ${ }^{1}$, Adriana Araceli Quiroga Salazar ${ }^{2}$, Flor Angélica Calcina Quispe ${ }^{3}$
\end{abstract}
\begin{abstract}
RESUMEN
Objetivo: Determinar el nivel de implicancias físicas y emocionales del profesional de Enfermería frente a la indicación médica del retiro de métodos de soporte vital en el paciente terminal de la Unidad de Cuidados Intensivos del Instituto Nacional Materno Perinatal y el Hospital Central de la Fuerza Aérea del Perú. Metodología: El diseño del estudio fue observacional, de tipo descriptivo y de corte transversal. La muestra fue universal, pues estuvo constituida por la población conformada por 30 enfermeros de las Unidades de Cuidados Intensivos, quienes participaron en forma voluntaria. Como instrumento, se utilizó un cuestionario elaborado por los investigadores, validado por juicio de expertos, que permitió recolectar datos específicos para evaluar el nivel de implicancias físicas y emocionales en los profesionales de Enfermería. Resultados: Los resultados obtenidos fueron: el $67 \%$ de los enfermeros presentó un nivel bajo de implicancias físicas y el $16.5 \%$, un nivel moderado; el 50\% presentó un nivel alto de implicancias emocionales y el $40 \%$ un nivel moderado. Conclusiones: La indicación de retiro de los métodos de soporte vital en el paciente terminal de la Unidad de Cuidados Intensivos tuvo un mayor nivel de implicancias emocionales y un menor nivel de implicancias físicas en los profesionales de Enfermería.
\end{abstract}

Palabras clave: Implicancias físicas, emocionales, indicación, retiro, soporte vital, paciente terminal.

\begin{abstract}
Objective: To determine the level of physical and emotional implications of the nursing professional medical advice against removal of life-sustaining terminal patient in the Intensive Care National Maternal Perinatal Institute and the Central Hospital of the Air Force Peru . Methodology: The study design was observational, descriptive, cross-sectional. The sample was universal, it consisted of the 30 nurses comprised of intensive care units who participated voluntarily population. As an instrument, a questionnaire developed by the researchers, validated by expert judgment, which allowed us to collect specific data to assess the level of physical and emotional implications nursing professionals was used. Results: The results were: $67 \%$ of nurses presented a low level of physical implications and $16.5 \%$ a moderate level; $50 \%$ had a high level of high emotional implications and $40 \%$ a moderate level. Conclusions: The indication of withdrawal of life support methods in the terminal patient ICU had a higher level of emotional implications and a lower level of physical implications for nurses.
\end{abstract}

Keywords: physical, emotional implications, indication, retirement, life support, terminal patient.

${ }^{1}$ Enfermera asistencial en el servicio de Cuidados Intensivos del Instituto Materno Perinatal, Lima

${ }^{2}$ Enfermera Jefe del Hospital Central Fuerza Aérea del Perú, Lima, Perú.

${ }^{3}$ Enfermera asistencial en el Instituto Nacional de Enfermedades Neoplásicas, Lima

Correspondencia: irene_1806@hotmail.com; adriacheli@hotmail.com 
Nivel de implicancias físicas y emocionales en el profesional de Enfermería frente a la indicación médica del retiro de métodos de soporte vital en el paciente terminal, de la Unidad de Cuidados Intensivos del "Instituto Nacional Materno Perinatal" y el "Hospital Central de la Fuerza Aérea del Perú", 2009.

\section{INTRODUCCIÓN}

La Unidad de Cuidados Intensivos es un servicio donde se atiende a pacientes en estado crítico, mayormente por problemas respiratorios, cardiovasculares, neurológicos, traumatismos encéfalo-craneanos, cáncer en fase terminal y sepsis que, debido a su complejidad y gravedad del cuadro clínico, la mayoría de los pacientes fallece (Ebias y Gómez, 2007).

Esta unidad cuenta con tecnología avanzada a fin de ayudar al paciente en su recuperación, sin embargo, esto lleva muchas veces solo a mantener las funciones vitales por largos períodos, sin llegar a curar o mejorar, al menos parcialmente, los procesos subyacentes que condujeron al paciente a esta situación. Por lo tanto, cuando no responde al tratamiento que se estableció en un plazo determinado o cuando el equipo identifica que la calidad de vida residual será inaceptable, se considera el retiro de las medidas de soporte vital (Gherardi, 2007).

La toma de decisión sobre el retiro de los métodos de soporte vital en un medio asistencial como la Unidad de Cuidados Intensivos es, en general, dependiente de la iniciativa médica, y se realiza cuando se evidencia ausencia de la respuesta en la sustitución de un órgano o la función, o cuando la presencia de irreversibilidad se manifiesta en el cuadro clínico por la sucesiva claudicación de órganos vitales (Gherardi, 1999).

Los enfermeros de la Unidad de Cuidados Intensivos poseen habilidades y destrezas que los capacitan para llevar a cabo diversos protocolos, sus habilidades se han tecnificado y su destreza se ha especializado para adelantarse a situaciones que comprometen la vida del paciente. Además, están llamados a asumir el papel protagonista en la etapa final de la vida del paciente, acompañando el proceso de la muerte o en las fases previas de la misma, momento donde la actitud de acogida, compañía y calor humano es más importante que en ningún otro (Reveron, 2006).

Sin embargo, cuando en la práctica se decide el retiro de determinados métodos de soporte vital en pacientes que están bajo su cuidado, se retiran angustiados y decepcionados después de haber trabajado concienzudamente por lograr su bienestar y supervivencia del paciente, con frecuencia experimentan un estado de frustración ante la muerte de uno de ellos y, en su ideal por salvar vidas, se culpan por no haber hecho más por él (Gálvez, Muñuvel, Del Águila, Ríos y Fernández 2007, Orgaz 1998).
Por ello, los profesionales de Enfermería, que trabajan con pacientes en etapa terminal, deben pactar previamente con sus sentimientos personales de impotencia y fracaso, aceptar sus limitaciones $\mathrm{y}$ aprender a reducir sus tensiones, pues si consiguen superar su crisis y tolerar esta situación, experimentarán un crecimiento maduro en el ámbito profesional y personal (Zax, 2006).

La interrogante surge luego de haber visto y experimentado situaciones al ejecutar la orden médica del retiro de método de soporte vital en el paciente terminal y el haber evidenciado las implicancias físicas y emocionales, en los profesionales de Enfermería que ejecutan dicha indicación, y también porque la gran mayoría de ellos refieren que se conmueven y piensan en su familia ante la muerte del paciente sujeto de su cuidado.

Por ello, el objetivo del presente trabajo es determinar el nivel de implicancias físicas y emocionales en los profesionales de Enfermería, frente a la indicación médica del retiro de métodos de soporte vital en el paciente terminal de la Unidad de Cuidados Intensivos del Instituto Nacional Materno Perinatal y del Hospital Central Fuerzas Armadas.

\section{MATERIAL Y MÉTODOS}

El diseño del estudio fue no experimental, de tipo descriptivo y de corte transversal, se realizó en el mes de mayo del 2009. El tipo de muestra fue universal, porque se incluyó a la población total conformada por 30 enfermeros de la Unidad de Cuidados Intensivos. Como instrumento de recolección de datos se utilizó un cuestionario elaborado por las investigadoras y validado por juicio de expertos, incluyendo datos generales y datos específicos con 4 ítems para evaluar el nivel de implicancias físicas y 7 ítems para evaluar el nivel de implicancias emocionales.

Para medir el nivel de implicancias físicas se utilizó la siguiente escala: nivel bajo de implicancia $=$ de 4 a 6 puntos, nivel moderado de implicancia $=$ de 7 a 9 puntos, nivel alto de implicancia $=$ de 10 a 12 puntos. Para medir el nivel de implicancias emocionales se utilizó la siguiente escala: nivel bajo de implicancia $=$ de 7 a 11 puntos, nivel moderado de implicancia $=$ de 12 a 16 puntos, nivel alto de implicancia $=$ de 17 a 21 puntos, cada respuesta de las escalas tiene un valor de 1 a 3 puntos. Donde 1 punto equivale a nunca, 2 puntos equivale a veces, 3 puntos equivale a siempre. 
El instrumento fue aplicado por las autoras en los diferentes turnos de los enfermeros que conformaron la muestra de estudio.

Para el procesamiento de los datos recolectados, se utilizó el programa SPSS 15.0.
Durante el desarrollo del estudio, se respetó la confidencialidad de los datos.

\section{RESULTADOS}

\section{Tabla 1}

Características sociodemográficas de los profesionales de Enfermería que laboran en la Unidad de Cuidados Intensivos del "Instituto Nacional Materno Perinatal” y el "Hospital Central de la Fuerza Aérea del Perú". Lima - Mayo, 2009.

\begin{tabular}{ccc}
\hline Edad & N. $^{\circ}$ & $\%$ \\
\hline 26 a 39 años & 18 & 60.00 \\
40 a 53 años & 12 & 40.00 \\
Tiempo de servicio en la UCl & $\mathrm{N}$ & $\%$ \\
1 a 5 años & 12 & 40.00 \\
6 a 10 años & 12 & 40.00 \\
11 a 15 años & 3 & 10.00 \\
16 a 20 años & 3 & 10.00 \\
Especialidad en UCl & $\mathrm{N}^{\circ}$ & $\%$ \\
No & 10 & 33.30 \\
Sí & 20 & 66.70 \\
Total & 30 & 100.00 \\
\hline
\end{tabular}

En la tabla 1 se observa que el $60 \%$ de la muestra que corresponde al grupo etario de 26 a 39 años y el $40 \%$ de 40 a 53 años. En relación al tiempo de servicio en la Unidad de Cuidados Intensivos, el
$80 \%$ tiene de 1 a 10 años de experiencia, y un $20 \%$ de 11 a 20 años. Finalmente el $66.7 \%$ cuenta con especialidad en UCI, y un $33.3 \%$ no cuenta con estudios de especialidad en el área de UCI.

Tabla 2

Nivel de implicancias físicas en el profesional de enfermería frente a la indicación médica del retiro del método de soporte vital en el paciente terminal de la Unidad de Cuidados Intensivos del "Instituto Nacional Materno Perinatal" y el "Hospital Central de la Fuerza Aérea del Perú”. Lima - Mayo, 2009.

\begin{tabular}{ccc}
\hline Implicancias físicas & N. ${ }^{\circ}$ & $\%$ \\
\hline Baja & 20 & 67.00 \\
Moderada & 5 & 16.50 \\
Alta & 5 & 16.50 \\
Total & 30 & 100.00 \\
\hline
\end{tabular}

En la tabla 2, se observa que el $67 \%$ de profesionales de Enfermería presentan un nivel bajo de implicancias físicas ante la indicación del retiro del soporte vital en el paciente terminal, y el 33\% un nivel de implicancias físicas entre moderado y alta. 
Nivel de implicancias físicas y emocionales en el profesional de Enfermería frente a la indicación médica del retiro de métodos de soporte vital en el paciente terminal, de la Unidad de Cuidados Intensivos del "Instituto Nacional Materno Perinatal" y el "Hospital Central de la Fuerza Aérea del Perú", 2009.

\section{Tabla 3}

Nivel de implicancias emocionales en el profesional de Enfermería frente a la indicación médica del retiro del método de soporte vital en el paciente terminal de la Unidad de Cuidados Intensivos del "Instituto Nacional Materno Perinatal" y el "Hospital Central de la Fuerza Aérea del Perú". Lima - Mayo, 2009.

\begin{tabular}{ccc}
\hline Implicancias emocionales & N. ${ }^{\circ}$ & $\%$ \\
\hline Baja & 3 & 10.00 \\
Moderada & 12 & 40.00 \\
Alta & 15 & 50.00 \\
Total & 30 & 100.00 \\
\hline
\end{tabular}

En la tabla 3 se observa que el $50 \%$ de los profesionales de Enfermería presenta un nivel alto de implicancias emocionales ante la indicación médica

\section{DISCUSIÓN}

En la primera tabla se observa que el mayor porcentaje de enfermeros presentó un nivel bajo de implicancias físicas, este indicador se vincula al grupo etareo de enfermeros encuestados que se encuentran entre las edades de 40 a 53 años, etapa en la que el ser humano ha obtenido niveles óptimos de madurez y desarrollo, lo que les permite afrontar los problemas con mayor serenidad y seguridad (Gómez, 2003). Sin embargo, también se observa un porcentaje significativo de implicancias físicas en un nivel moderado y alto, estos resultados son corroborados por (Moran, 2004) quien refiere que toda reacción emocional es subjetiva y personal, es decir que cada individuo experimenta una emoción de forma particular dependiendo de su experiencia y situación concreta y las reacciones fisiológicas que se pueden experimentar con mayor probabilidad son el aumento del ritmo respiratorio, aumento del ritmo cardíaco, sudoración, en las manos. Los profesionales de Enfermería del presente estudio, ante la indicación del retiro de método de soporte vital del paciente terminal, no están ajenos de evidenciar dicha reacciones físicas.

En la segunda tabla se observa que el mayor porcentaje de enfermeros presentó un nivel alto de implicancias emocionales probablemente porque la mayoría de ellos tienen menos años de experiencia laboral. El profesional de enfermería que labora en la Unidad de Cuidados Intensivos es la persona que llega a conocer y detectar, con mayor acierto, las necesidades e inquietudes del paciente, su relación es íntima de contacto físico y emocional (Padilla y Silva, 2007) y que, además, es la persona que acompaña en el proceso de muerte o en las fases del retiro de método de soporte vital en el paciente terminal, un $40 \%$ presenta un nivel moderado, y un $10 \%$ presenta un nivel bajo.

previas de la misma, momento donde la actitud de acogida, compañía y calor humano es muy importante (Orgaz, 1998).

Por ello, el acercamiento a la muerte genera emociones y sentimientos de difícil manejo para muchos de los profesionales de Enfermería despertando inquietud, ansiedad e impotencia, al involucrarse con el moribundo: primero, porque la muerte del paciente los hace tomar conciencia de su propia mortalidad y, en segundo lugar, es quien más horas pasa al lado del paciente $\mathrm{y}$, al verlo morir, les invade sentimientos de impotencia por no haber hecho más por el paciente (Colell, Limonero y Dolores 2003 2003).

Es importante que el profesional de Enfermería consiga superar la crisis emocional para seguir atendiendo a los pacientes. De lo contrario terminarán con manifestaciones de ansiedad y estrés, llegando a una situación de agotamiento emocional, produciéndose distanciamiento con el paciente, con el respectivo deterioro de las relaciones interpersonales como consecuencia negativa, tanto para el paciente como para el enfermero, al estar incapacitado emocionalmente para desarrollar su labor (Colell, Limonero y Dolores 2003).

El profesional de enfermería que labora en áreas críticas, se enfrenta con frecuencia a situaciones difíciles que afectan su estado emocional, por tanto, debe estar preparado para manejar sus emociones de manera que no obstaculicen su forma de razonar y poder tomar decisiones acertadas de acuerdo con sus valores, normas sociales y culturales (Mora, 2008). Esto, contribuirá a mantener su bienestar y el de su entorno. 
Se concluye que la indicación de retiro del soporte vital en los pacientes terminales en la UCI, ocasiona implicancias físicas y emocionales en el profesional de enfermería, siendo las implicancias emocionales las que alcanzan un alto nivel en el grupo de estudio.

\section{REFERENCIAS BIBLIOGRÁFICAS}

Berner, J. (1987). El quehacer de enfermería ante el paciente al final de la vida. Madrid: Editorial San Pablo.

Colell, R., Limonero, J., y Dolores, O. (2003). Actitudes y emociones en estudiantes de enfermería ante la muerte y la enfermedad terminal. Investigación en salud. 5(2), 1405 -7980. Recuperado en: http://www.redalyc.org/ articulo.oa?id=14250205

Ebias, A. (2007). Cuidados Intensivos. Estados Unidos: Editorial Glass.

Guevara, B. (2007). Perfil de la enfermera intensivista. España: Editorial Interamericana McGraw Hill.

Gómez, E. (2003). Adulto maduro. México: Editorial Silva.

Gherardi, C. (2007). Vida y muerte en terapia Intensiva. Estrategias para conocer y participar en las decisiones. Buenos Aires: Editorial Biblos.

Gherardi, C. et al (2002). La muerte intervenida. De la muerte cerebral a la abstención o retiro del soporte vital. Medicina. 66(3), 3-5.
Gálvez, M., Muñumel, A., Del Águila, B., Ríos, F., Fernández, M. (2007). Vivencias y perspectivas de la enfermera en el proceso de la muerte en UCI. España: Biblioteca las Casas.

Mora, C. (2008). Saber vivir manejando las emociones. México: Copyright

Moran, R. (2004). Educando con desórdenes emocionales $y$ conductuales. Puerto Rico: Editorial Universidad Puerto Rico.

Orgaz, J. (1998). La muerte. España: Editorial Salvat.

Padilla, D. y Silva, S. (2007). Dilema ético en el profesional de Enfermería en unidades de cuidado intensivo. Revista de Enfermería. 10(2), 20-1. Recuperado en: http://www.encolombia.com/medicina/enfermeria/ revista10_2_2007/Dilemas_y_Toma_de cisiones.htm.

Reverón, D. (2006). Que hacer de la enfermera en el Paciente Terminal. España: Editorial Doyma.

Zax, M. (2006). Paciente terminal. España: Editorial La Senda Copyright. 